\title{
Evaluation and Outcome of Patients of Stemi with Acute Total Occlution of Coronary Artery in The Setting of Primary PCI, Pharmaco Invasive PCI and Delayed PCI
}

\author{
Kaushik Paul, Sudeb Mukherjee*, Saumyajit Ghosh and SC Mandal \\ Department of Cardiology, ICVS, IPGME\&R, India
}

Submission: September 18, 2018; Published: December 12, 2018

*Corresponding author: Sudeb Mukherjee, Department of Cardiology, ICVS, IPGME\&R, 4/28 A, Jadavgarh, Dr. BC Roy Road, Kolkata-700078, West Bengal, India

Abstract

Introduction: Better medical facilities have come up and coronary interventions and cardiac surgeries are being performed in nearly all major Indian cities. Still a vast number of patients are not able to get the benefit of primary Percutaneous Coronary Intervention (PCI). Most of them can be managed with pharmacoinvasive or delayed PCI, both of which can be beneficial in the long term from prognostic point of view.

Objectives: The present study was therefore carried out to evaluate and compare patients of STEMI with acute total occlusion of coronary artery in the setting of Primary PCI, PharmacoInvase PCI and Delayed PCI in terms of Left Ventricular Ejection Fraction (LVEF) and mortality at before discharge at 1 month and 6 months.

Methods: A total of 33,30and 31in the three different categories of PCI patients were studied in detail and analysed accordingly.

Results: Majority of patients have almost same epedemilogical background. In most of the patients (71.4\%) Ejection Fraction was between 35-53\%. Regarding Ejection fraction fraction improvement Primary PCI showed significant improvement in when preintervention Ejection fraction was compared with Post intervention Ejection Function just before discharge, at 1 month and 6 months. In Pharmacoinvasive PCI group there was no significant improvement when preintervention Ejection fraction was compared with Post intervention Ejection Function just before discharge but showed significant improvement at 1 month and 6 months. In Delayed PCI group there was no significant improvement when preintervention Ejection fraction was compared with Post intervention Ejection Function just before discharge but showed significant improvement at 1 month and 6 months. Regarding mortality Primary PCI had more no. of mortality compared with the other two groups.

Keywords: Primary Percutaneous Coronary Intervention (PPCI); Pharmaco invasive PCI; Delayed PCI; Left Ventricular Ejection Fraction (LVEF)

\section{Introduction}

At the most severe end of the spectrum of acute coronary syndromes is ST-Segment Elevation Myocardial Infarction (STEMI), which usually occurs when a fibrin-rich thrombus completely occludes an epicardial coronary artery. The diagnosis of STEMI is based on clinical characteristics and persistent ST-segment elevation as demonstrated by 12-lead electrocardiography. Patients with STEMI should undergo rapid assessment for reperfusion therapy, and a reperfusion strategy should be implemented promptly after the patient's contact with the health care system. Two methods are currently available for establishing timely coronary reperfusion: primary percutaneous coronary intervention and fibrinolytic therapy [1]. Percutaneous coronary intervention is the preferred method but is not always available. ST-segment elevation myocardial infarction (STEMI) is characterized by total occlusion of the infarct-related artery in contrast to Unstable Angina or Non-ST elevate d Myocardial
Infarction (UA/NSTEMI) [2]. Evidence from several randomized clinical trials during the past two decades has established the importance of the open artery theory, which states that prompt and complete restoration of flow in the occluded artery decreases infarct size, preserves Left Ventricular (LV) function, and improves survival rates. The role of Percutaneous Coronary Interventions (PCIs) in the early hours of an STEMI can be divided into primary PCI, Pharmacoinvasive PCI, and Delayed PCI [3].

Primary PCI can be defined as coronary angioplasty/stenting without prior administration of fibrinolytic agents or GPIIb/ IIIa antagonists. A pharmaco-invasive strategy can be defined as pharmacological reperfusion (using fibrinolytic agents) with an 'invasive back-up', which means that patients are transported to a PCI hospital for either immediate rescue PCI in case of failed fibrinolysis or nonurgent coronary angiography to determine the need for additional treatment of the culprit lesion (PCI or bypass 
surgery). This strategy has been shown to be superior to a very conservative approach of in-hospital fibrinolysis with transfer to a PCI centre only in case of failed thrombolysis. Delayed PCI of the infarct artery is performed in patients treated with an initial noninvasive strategy (i.e., with fibrinolysis or without reperfusion therapy) who become unstable because of the development of cardiogenic shock, acute severe heart failure, or unstable post infarction angina, provided that invasive management is not considered futile or inappropriate [4]. Delayed PCI also encompasses interventions performed for fibrinolytic failure [5] or infarct artery re-occlusion, as part of an invasive strategy for patients after successful fibrinolysis, and for patients who did not receive reperfusion therapy but who did demonstrate significant residual ischemia during hospitalization. Primary PCI of the infarct artery is preferred to fibrinolytic therapy when time-to-treatment delays are short and the patient presents to a high-volume, wellequipped center with experienced interventional cardiologists and skilled support staff. Compared with fibrinolytic therapy, primary PCI produces higher rates of infarct artery patency, TIMI 3 flow, and access site bleeding and lower rates of recurrent ischemia, reinfarction, emergency repeat revascularization procedures, intracranial hemorrhage (ICH), and death [6].

Primary PCI within the recommended guidelines time window cannot be offered to all patients, not even with a well-functioning network of ambulances and hospitals. For some of these patients, especially those presenting very early without an increased risk of bleeding, immediate lytic therapy (in the ambulance or the emergency department of the community hospital) is still the best treatment, provided they can be transferred to a PCI hospital for rescue $\mathrm{PCI}$, or for angiography, in order to decide on final treatment of the culprit lesion (PCI, bypass surgery or in some cases no mechanical treatment). Ideally, these patients should be transported to the PCI hospital immediately after starting lytic therapy. On arrival at the PCI hospital, a new ECG should be taken, and the decision made to perform angiography either immediately or within 24 hrs. Even in patients who has Cardiogenic shock or acute severe HF that develops after initial presentation

Keeping all the above-mentioned guidelines in mind our objective is to evaluate and find out the outcome in all these various group of patients who are undergoing different types of procedure which are best in their time frame at initial presentation, after one month and after 6 months of follow up.

\section{Aims \& Objectives}

a. To evaluate Post PCI and left ventricular ejection fraction at Baseline, 30 days and 6 months

b. To observe Post PCI and 30 day and 6 months mortality.

\section{Materials and Methods}

Study setting: The study was conducted at the Department of Cardiology, IPGME\&R, S.S.K.M. Hospital, Kolkata.

Study period: Study was conducted from November 2016 to October 2017.
Study design: Hospital based Prospective type of study (observational cohort study).

Study population: The study included a consecutive series of 33 patients with acute STEMI who presented within the first $24 \mathrm{~h}$ after symptom onset who were treated with Primary PCI between November 2015 and October 2016, 30 patients who were treated with Pharmacoinvasive PCI and 31 patients who were treated with Delayed PCI.

Inclusion Criteria: 1) Clinical symptoms of MI. 2) Either $\geq 1$ mm ST Elevetion in 2 contiguous leads with acute total occlusion on angiography.

\section{Data Collection}

The clinical characteristics, electrocardiographic parameters, angiographic and procedural was collected using a pre-designed, pre-tested and semi structured schedule by the investigator himself. Informed consent was taken from all the patients.

Echocardiography was done before, and at the time of discharge, 30 days after and at 6 months. Primary objective is to evaluate the Left Ventricular function at onset, at the time of discharge after PCI, 1 month after PCI and 6 months after PCI and mortality was observed Post PCI, 1 month and 6 months after procedure.

\section{End Points and Follow-Up}

The primary end point of the study was comparison of Ejection Fraction in the 3 groups and occurrence of death from any cause. The follow-up information was collected by a phone call/outpatient visit till 6 months. Patients who had cardiac complaints underwent complete clinical, ECG, and laboratory evaluation. Information on deaths was obtained from hospital records, death certificates or phone contact with relatives of the patient or the patient's referring physician.

\section{Data Analysis}

All categorical variables are depicted using relative frequency distributions. Continuous data are presented as the mean \pm SD, the median (with 25th-75th percentiles) or counts and proportions (percentages) as appropriate. The normality of data distribution was analyzed using a Kolmogorov-Smirnov test. Normally distributed, continuous variables are expressed as means $( \pm \mathrm{SD})$, while other continuous data are expressed as median with Inter Quartile Range (IQR). Categorical variables were compared using the chi-square statistics or Fisher's exact test for, while the 1-way ANOVA or Kruskal-Wallis test was used for continuous and ordinal variables, as appropriate. The following variables were included in the model: age, gender, Killip class (III-IV vs I-II), preprocedural TIMI flow (3 vs 0,1 , or 2), postprocedural TIMI flow, anterior location, total ischemic time, and diabetes. $\beta$ coefficient was calculated to explain the variation in dependent variable by independent variables. Independent predictors of mortality were analyzed using multivariate logistic regression analysis. Odds ratio was calculated to the following variables were included in the model: age, gender, Killip class, postprocedural TIMI flow, 


\section{Journal of Cardiology \& Cardiovascular Therapy}

and risk factors. Event rates were determined and displayed with Kaplan-Meier methodology and compared with the log-rank test. Statistical analyses were performed using Statistical Package for Social Sciences, version 20.0and Stata/SE, version 9.2Texas). p Values $<0.05$ were considered statistically significant.

\section{Results and Analysis}

\section{Study cohort}

A total of 36 STEMI patients underwent primary PCI at our institution between 2016- 2017. We excluded 2 patients who underwent primary PCI involving left main coronary artery or a bypass graft and 1 patient because of missing ECG recordings. Total 33 patients were included of which 3 patients died after procedure before discharge and ejection fraction could not be compared post procedure before discharge, at 1 month and 6 months post discharge. In the pharmacoinvasive group 30 patients were eligible for the study and recruited. In the delayed PCI group also 31 patients were eligible for the study and recruited.

\section{Descriptive Analysis}

A total of 33 patients were studied in primary PCI group. The minimum and maximum value for age was 45 and 69 years. The mean age is 59.21 with standard deviation of 5.8. In Pharmacoinvasive group minimum value for age was 42 and maximum was 71 . The mean age was 56.43 with a standard deviation of 7.22. In delayed PCI group minimum age was 48 and maximum was 68 years, the mean age was 59.03 with a standard deviation of 6.24. The difference of mean between the three group was not statistically significant with a p value of 0.74 .

87.9\% of the patient in primary PCI group was male and 12.15 were female. In the pharmacoinvasive and delayed group male and female percentage were $80 \%: 20 \%$ and $83.9 \% 16.1 \%$ respectively. Symptomatically almost all presented with severe chest pain.

Almost all the patients had some past history: majority had Smoking followed by hypertension, Diabetes Mellitus. 1/7th (15.2\%) of patients had positive family $\mathrm{H} / \mathrm{O}$ cardiovascular disTable 2: Showing results of LVEF in PPCI group $(n=30)$.

\begin{tabular}{|c|c|c|c|}
\hline & N & Mean Std. Error Mean & Std. Deviation \\
\hline PreIntervention PostIntervention_before_Discharge & 30 & 42.6 & 5.3794 \\
\cline { 2 - 5 } & 30 & 46.7667 & 5.81867 \\
\hline PostIntervention_After_1_month & 30 & 52.1333 & 5.30279 \\
\hline PostIntervention_after_6_month & 30 & 54.8 & 5.99655 \\
\hline
\end{tabular}

Table 3: Showing results of LVEF in Pharmacoinvasive group in different time periods $(n=30)$.

\begin{tabular}{|c|c|c|c|c|}
\hline & $\mathbf{N}$ & Mean & Std. Deviation & Std. Error Mean \\
\hline PostIntervention_After_1_month & 30 & 52.8667 & 6.42588 & 1.1732 \\
\hline PostIntervention_after_6_month & 30 & 54.2333 & 7.15196 & 1.30576 \\
\hline \multirow{2}{*}{ PreIntervention PostIntervention_before_Discharge } & 30 & 48.3667 & 5.38506 & 0.98317 \\
\hline & 30 & 51.0667 & 5.65035 & 1.03161 \\
\hline
\end{tabular}

When Ejection Fraction was compared in Pharmacoinvasive Group in different time period the following results were obtained (Table 3). Comparison between PreIntervention EF and Post ease. Of the all study subjects, $50-80 \%$ had anterior wall MI if all the three groups are considered. Distribution of subjects according to CAG findings (\%) has been shown in Table 1.

Table 1: Showing distribution of Angiographic pattern in all the groups.

\begin{tabular}{|c|c|c|c|}
\hline & Primary & Pharmacoinvasive & Delayed \\
\hline LAD & 44.64286 & 47.91666 & 47.5 \\
\hline LCX & 26.78571 & 20.83333 & 22.5 \\
\hline RCA & 28.57143 & 31.25 & 30 \\
\hline
\end{tabular}

When comparing LVIDD in between the different groups shows the following results. In the PPCI group $(n=33)$ the mean was $48.63 \mathrm{~mm}$ (SD-2.70, SE-0.47); pharmacoinvasive group $(\mathrm{n}=30)$ the mean was $47.06 \mathrm{~mm}$ (SD-6.23. SE-1.13) and in the Delayed PCI (31) group mean was 50.87(SD-5.71, SE-1.02).

When comparing LVIDS in between the different groups the results were with mean value of $37.24 \mathrm{~mm}$ (SD-3.25, AE-0,56), $34.33 \mathrm{~mm}$ (SD-5.81, SE 1.06) and 38.67mm (SD-4.96, SE-0.89) in the PPCI, pharmacoinvasive and Delayed respectively.

When comparing the Ejection Fraction between different groups before intervention, revealed mean value of $41.78 \%$ (SD-5.81, SE-1.01) in the PPCI group. For pharmocoinvasive and delayed group it was $48.43 \%$ (SD-5.44, SE-1.01), and $46.93 \%$ (SD6.21 , SE-1.11) respectively.

When comparing the Ejection Fraction in Primary PCI in different time frame the following values were obtained (Table 2). Comparison between PreIntervention EF and Post intervention EF before discharge in Primary PCI group revealed improvement from mean of $42.60 \%$ to $46.76 \%$ with statistical significant $p$ value of 0.0025 . at 1 month mean EF was 52.13 (p value 0.0001 ) and at after 6 months EF was 54.80 with p value 0.0001 compared to pre-interventional value. However, Comparison between Post intervention EF after 1 month of discharge and Post intervention EF after 6 months of discharge did not reveal statistical significant value. intervention EF before discharge revealed improvement in EF from $48.36 \%$ to $51.06 \%$ with $p$ value of 0.06 . At 1 month the LVEF was $52.86 \%$ ( $p$ value of 0.0047 ) and at 6 months $54.23 \%$ with $p$ 
value of 0.0007. Comparison between Post intervention EF before discharge and Post intervention EF after1 month of discharge did not reveal significant value $(p=0.2540)$ and at 6 months $(\mathrm{p}=0.0620)$.

Table 4: Showing results of LVEF in Delayed $\mathrm{PCl}$ group in different time period $(n=31)$.

\begin{tabular}{|c|c|c|c|}
\hline & N & Mean & Std. Deviation \\
\hline PreIntervention & 31 & 46.9355 & 6.21254 \\
\hline PostIntervention_before_Discharge & 31 & 49.3548 & 5.78244 \\
\hline After_3_Months & 31 & 52.129 & 5.70229 \\
\hline After_6_Months & 31 & 54.6452 & 6.55514 \\
\hline
\end{tabular}

When comparing the Ejection fraction in Delayed PCI (Table 4) LVEF improvement was not statistically significant between PreIntervention EF and Post intervention EF before discharge. ( $46.93 \%$ to $49.35 \%$ with $p$ value of 0.1177 ). However, at 1 month and 6 months the improvement was significant with $p$ value of 0.0011 and at 6 months $\mathrm{p}=0.0001$ respectively.

Comparison between Post intervention EF before discharge and Post intervention EF after 1 month of discharge did not reveal any significant improvement $(\mathrm{p}=0.062)$. Comparison between Post intervention EF before discharge and Post intervention EF after 6 month of discharge revealed improvement with significant $\mathrm{p}$ value of 0.0013 .

\section{Analysis of Survival using Kaplan-Meier Method}

Kaplan-Meier survival curves was drawn for Ejection Fraction. Quantitative variables were transformed into categorical variables, as appropriate.

Kaplan-Meier Survival Probability analysis was made to estimate time-to-Death and compare survival experiences of different groups. The lengths of the horizontal lines along the $\mathrm{X}$-axis of serial times represent the survival duration for that interval. The interval is terminated by the occurrence of Death. The cumulative probability of surviving a given time is seen on the Y-axis. The vertical distances between horizontals illustrate the change in cumulative probability as the curve advances.

\section{Comparison of Survival curves}

The survival curves were compared statistically by testing the null hypothesis i.e. there is no difference regarding survival among groups. This null hypothesis was statistically tested by another test known as log-rank test and Cox proportion hazard test In log-rank test we calculated the expected number of death in each. The test calculates the chi-square (X2) for each event time for each group and sums the results. The summed results for each group are added to derive the ultimate chi-square to compare the full curves of each group Comparison of survival curves by log rank test found that though Death occurred in Primary PCI group, difference was not statistically significant for Ejection Fraction (Figure 1).

\section{Survival proportions: Survival of Data 1}

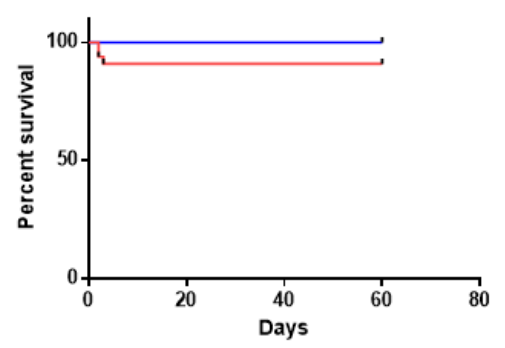

- Primary - Pharmacoinvasive

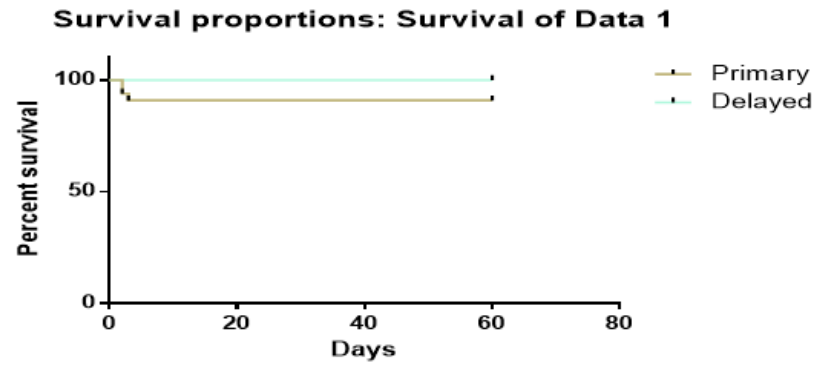

Figure 1: Showing comparison of survival benefit in PPCI Versus Pharmacoinvasive and PPCI Versus Delayed group.

\section{Discussion}

In our study we found that majority $(97 \%, 96.7 \%, 80.6 \%)$ had Smoking followed by hypertension $(60.6 \%, 26 \%, 41.9 \%)$, Diabetes Mellitus $(21.2 \%, 6.7 \%, 6.5 \%)$ in each group of PPCI, Pharmacoinvasive and Delayed PCI respectively. In Indian subcontinent percentage of smokers are quite high and this has been reflected in our data. 1/5th (17.9\%) of patients had positive family $\mathrm{H} / \mathrm{O}$ cardiovascular disease. This high prevalence of hypertension, Diabetes positive in the study group are in accordance with the risk factors of AMI. The prevalence of diabetes has increased in last two decades and India has earned the dubious distinction of becoming the world's capital of coronary heart disease and diabetes. There was statistical significant difference between mean systolic BP when Primary and Pharmacoinvasive group, Pharmacoinvasive and Delayed PCI group were compared but not between Primary and Delayed group. Mean BMI came out to be 25, 24.5, 25.77 respectively. For many Asian populations, additional trigger points of BMI for public health action were identified, and in this study, more than half (55.4\%) of the patients falls in the category of increased risk of co-morbid conditions.

In the present study, there were more patients with Anterior wall MI $(78.78 \%, 76.66 \%, 54.83 \%)$ as compared to Inferior wall (21.21\%,23.33\%,45.16\%). Gjin Ndrepepa et al also had Similar findings in their study (Anteriorwall MI-61.6\%). Main culpritvessel was Left Anterior Descending Artery (44.64\%,47.92\%,47.5\%) followed by Right Coronary Artery $(28.78 \%, 20.88 \%, 22.5 \%)$ and 
Left Circumflex artery $(28.57 \%, 31.25 \%, 30 \%$ respectively there was no significant difference in TIMI Score in between the three groups. Majority of patients were in Killip Class I and II.

Mean LVEF in each individual group before and after intervention has been depicted in (Table 1-3). Similar findings were seen in a study conducted by Kaul U et al. [7], they found that young Indian patients with MI and obstructive CAD have a high frequency of coronary risk factors, especially smoking and severe multiple-vessel disease.

When comparing the ejection fraction in primary PCI group and all the four point of time was considered then $P$ value was found to be statistically significant showing that Primary PCI had significant benefit as per Ejection fraction is considered. Comparison between Preintervention group and Post Intervention group before discharge at one month and at 6 month showed significant benefit with $\mathrm{P}$ value of $0.0025, \mathrm{P}<0,0001)$, $(\mathrm{P}<0,0001)$. Comparison between Post Intervention group before discharge and after 1 month of discharge and at 6 months also found statistically significant benefit with $P$ value 0,0001 . But there was no statically significant benefit with $\mathrm{p}$ value of 0.07 when improvement from 1 month to 6 months is considered.

When comparing the ejection fraction in Pharmacoinvasive PCI group and all the four point of time was considered then $P$ value was found to be statistically significant with $P$ value of 0.063. When Preintervention Group was compared with PostIntervention after 1 month $(\mathrm{P}<0,0047)$ after $6(\mathrm{P}<0,0007)$.

When comparing the ejection fraction in Delayed PCI group and all the four point of time was considered then $P$ value was found to be statistically significant.

When primary PCI is compared with Pharmacoinvasive PCI statically significance was found in Preintervention $(\mathrm{P}<0.0001)$ group and Post Intervention group $(\mathrm{P}<0.0004)$ before discharge but not between Post Intervention Group at 1 month $(\mathrm{P}=0.63)$ and at 6 month $(\mathrm{P}=0.74)$.Our study correlate well with study performed by TimoBaks, Robert-Janvan Geuns et.al who found a marked increase in overall mean EF was observed from $48 \pm 11 \%$ at baseline to $55 \pm 9 \%$ at follow-up [8].

The main endpoint of the current study was the occurrence of death during a 6 months follow-up after Primary PCI, Pharmacoinvasive PCI and Delayed PCI. Among Primary PCI group there was initially 33 patients 3 Patient died Predischarge although there was no evidence of death after discharge in Primary PCI group over 6 months follow up. In PharmacoInvasive PCI there was no death post PCI over 6 months follow up. In delayed PCI also there was no death Post PCI over a period of 6 month. Cardiac event occurred in males more than females and who had risk factors like smoking, diabetes or hypertension and anterior wall MI with low ejection fraction.

\section{Summary}

In recent years, significant changes have occurred in the strategy of reperfusion therapy in patients with STEMI that have included a more liberal use of Primary PCI, the routine use of stents and glycoprotein IIb/ IIIa inhibitors, the creation of integrated networks for the treatment of STEMI, and the extension of revascularization procedures to a broader population who present with a greater clinical risk profile than previously [9-11]. Several data indicate that the main determinant of the recovery of LV function [12] and ultimately of the prognosis [13] in patients. However, primary PCI is performed at less than $25 \%$ of acute care hospitals even in the United States $[14,15]$.

Even as CVD rates skyrocket, the availability of better cardiology facilities and dedicated cardiac centres have come as a welcome relief and India has seen a big leap in the fields of interventional cardiology and cardiac surgery in recent times [7].

Many patients with myocardial infarction with ST-segment elevation present to hospitals that do not have the capability of performing PCI and therefore cannot undergo PCI within the timelines recommended in the guidelines [16] instead, they receive fibrinolysis as the initial reperfusion therapy.

A mounting body of evidence suggests that delayed stent implantation is also helpful to patients as adjunctive anticoagulation and antiplatelet therapy have allowed thrombus burden meltdown $[17,18]$.

This study showed the same kind of predilection that pharmacoinvasive and Delayed PCI approach where applicable can be of greater help considering the improvement of $\mathrm{LV}$ function.

\section{Limitations}

This research was conducted only on a small size of population who had STEMI. A larger study population is required to confirm the relative prognostic importance.

\section{References}

1. Silber S, Albertsson P, Aviles FF, Camici PG, Colombo A, et al. (2005) Guidelines for percutaneous coronary interventions. The Task Force for Percutaneous Coronary Interventions of the European Society of Cardiology. Eur Heart J 26(8): 804-847.

2. Keeley EC, Boura JA, Grines CL (2003) Primary angioplasty versu intravenous thrombolytic therapy for acute myocardial infarction: a quantitative review of 23 randomised trials. Lancet 361(9351): 13-20.

3. White HD, Assmann SF, Sanborn TA, Jacobs AK, Webb JG, et al. (2005) Comparison of percutaneous coronary intervention and coronary artery bypass grafting after acute myocardial infarction complicated by cardiogenic shock: results from the Should We Emergently Revascularize Occluded Coronaries for Cardiogenic Shock (SHOCK) trial. Circulation 112(13): 1992-2001.

4. Newby LK, Hasselblad V, Armstrong PW, Van de Werf F, Mark DB, et al. (2003) Time-based risk assessment after myocardial infarction. Implications for timing of discharge and applications to medical decision-making. Eur Heart J 24(2): 182-189.

5. Gershlick AH, Stephens-Lloyd A, Hughes S, Abrams KR, Stevens SE, et al. (2005) Rescue angioplasty after failed thrombolytic therapy for acute myocardial infarction. N Engl J Med 353(26): 2758-2768.

6. Wijeysundera HC, Vijayaraghavan R, Nallamothu BK, Foody JM, Krumholz HM, et al. (2007) Rescue angioplasty or repeat fibrinolysis after failed fibrinolytic therapy for STsegment myocardial infarction: a meta-analysis of randomized trials. J Am Coll Cardiol 49(4): 422-430. 
7. Kaul U, Bhatia V (2010) Perspective on coronary interventions \& cardiac surgeries in India. Indian J Med Res 132: 543-548.

8. Baks T, Van Geuns RJ, Biagini E, Wielopolski P, Mollet NR, et al. (2005) Recovery of left ventricular function after primary angioplasty for acute myocardial infarction. Eur Heart J 26(11): 1070-1077.

9. Kondo M, Nakano A, Saito D, Shimono Y (1998) Assessment of microvascular no-reflow phenomenon using technetium-99 m macroaggregated albumin scintigraphy in patients with acute myocardial infarction. J Am Coll Cardiol 32(4): 898-903.

10. Maes A, Van de Werf F, Nuyts J, Bormans G, Desmet W, et al. (1995) Impaired myocardial tissue perfusion early after successful thrombolysis: impact on myocardial flow, metabolism, and function at late follow-up. Circulation 92(8): 2072-2078.

11. Agati L, Voci P, Hickle P, Vizza DC, Autore C, et al. (1998) Tissue-type plasminogen activator versus primary coronary angioplasty: impact on myocardial tissue perfusion and regional function 1 month after uncomplicated myocardial infarction. J Am Coll Cardiol 31(2): 338343.

12. Ito H, Okamura A, Iwakura K, Masuyama T, Hori M, et al. (1996) Myocardial perfusion patterns related to thrombolysis in myocardial infarction perfusion grades after coronary angioplasty in patients with acute anterior wall myocardial infarction. Circulation 93(11): 19931999.

13. Ito H, Maruyama A, Iwakura K, Takiuchi S, Masuyama T, et al. (1996) Clinical implication of the "no reflow" phenomenon: a predictor of

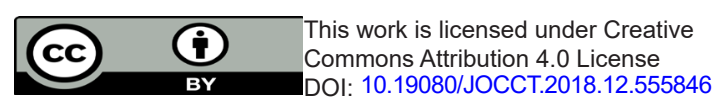

complication and left ventricular remodeling in reperfused anterior wall myocardial infarction. Circulation 93(2): 223-228.

14. Nallamothu BK, Bates ER, Herrin J, Wang Y, Bradley EH, et al. (2005) Times to treatment in transfer patients undergoing primary percutaneous coronary intervention in the United States: National Registry of Myocardial Infarction (NRMI)-3/4 analysis. Circulation 111: 761-767.

15. Bates ER, Nallamothu BK (2008) Commentary: the role of percutaneous coronary intervention in ST-segment-elevation myocardial infarction. Circulation 118(5): 567-573.

16. Antman EM, Hand M, Armstrong PW, Bates ER, Green LA, et al. (2008) 2007 Focused update of the ACC/AHA 2004 guidelines for the management of patients with ST-elevation myocardial infarction: a report of the American College of Cardiology/American Heart Association Task Force on Practice Guidelines. J Am Coll Cardiol 51(2): 210-247.

17. Jolicoeur EM, Tanguay JF (2011) From primary to secondary percutaneous coronary intervention: the emerging concept of early mechanical reperfusion with delayed facilitated stenting-when earlier may not be better. Can J Cardiol 27(5): 529-533.

18. Tang L, Zhou SH, Hu XQ, Fang ZF, Shen XQ (2011) Effect of delayed vs immediate stent implantation on myocardial perfusion and cardiac function in patients with ST-segment elevation myocardial infarction undergoing primary percutaneous intervention with thrombus aspiration. Can J Cardiol 27(5): 541-547.

\begin{tabular}{l} 
Your next submission with Juniper Publishers \\
will reach you the below assets \\
- Quality Editorial service \\
- Swift Peer Review \\
- Reprints availability \\
- E-prints Service \\
- Manuscript Podcast for convenient understanding \\
- Global attainment for your research \\
- Manuscript accessibility in different formats \\
( Pdf, E-pub, Full Text, Audio) \\
- Unceasing customer service \\
Track the below URL for one-step submission \\
https://juniperpublishers.com/online-submission.php \\
\hline
\end{tabular}

